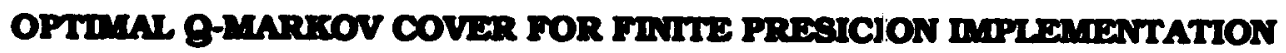

By

\author{
Darrell Williamson \\ ACT 2601. Australla \\ and \\ Robert E. Skelton \\ Purdue University \\ West Lafayette. Indiana
}

Australian National Untversity Canberra

\begin{abstract}
The existing q-Markov COVER realization theory does not take into account the problems of anthmetic errors due to both the quantization of states and coeficients of the reduced order model. All q-Markov COVERs allow some freedom in the choice of parameters. In this talk, we exploit this freedom in the exdsting theory to optimize the models with respect to these inite wordlength effects.
\end{abstract}




\title{
Optimal q-Markov Cover for \\ Finite Precision Implementation
}

\section{Darrell Williamson* and Robert E. Skeltor ${ }^{* *}$}

\begin{abstract}
The existing q-Markov COVER realization theory does not take into account the problems of arithmetic errors due to both the quantization of states and coefficients of the reduced order model. All q-Markov CCIVERs allow some freedom in the choice of parameters. In this paper we exploit this freedom in the existing theory to optimize the models with respect to these finite wordlength effects.
\end{abstract}

*Dept. of Systems Engineering, Research School of Physical Sciences, Australian National University Canberra, ACT 2601, Australia

** School of Aeronautics and Astronautics, Purdue University, West Lafayette, IN 47907, U.S.A. 


\section{Introduction}

An asymptotically stable system can be characterized in terms of its impulse response sequence (Markov parameters) and its output covariance sequence (covariance parameters) due to a zero mean white noise input process. A general approach has been developed [3] for realizing a system which matches q Markov parameters and $q$ covariance parameters. Such a system is referred to as a qMarkov COVER, and q-Markov COVERs may be generated from output data $[3,4]$ or from higher order models [5,6]. The Markov and covariance parameters are not independent and consequently the q-Markov COVER is not unique. In particular, all q-Markov COVERs are not related by state space similarity transformations [4]. In this paper we shall exploit the remaining degrees of freedom to optimize the q-Markov COVER realization with respect to an aspect of its finite wordlength realization.

Specifically, when digital controllers are to be implemented, both the controller coefficients and the controller states must be represented in finite wordlength precision. This finite wordlength (FWL) representation (or quantization) causes inaccuracies in the response when compared to the ideal (i.e. infinite precision) behaviour. Effects of quantization on the controller are increased noise at the output due to internal state quantization, and errors in time and frequency response characteristics due to coefficient errors.

In digital filter design, the FWL effects are known to be most significant when the poles of the filter are very close to the unit circle [12]. In particular, narrow band filters have all these poles near $z=1 \pm$ jo. For digital control, the zero-order-hold equivalent of a continuous time model (or controller) with a pole at $\lambda$ will have a discrete pole at $\exp (\lambda T)$. Hence for fast sampling and/or low damping of the continuous models, the discrete model will behave like a narrow band filter. The synthesis of optimal digital controllers with respect to arithemetic quantization noise is an important consideration in design especially for continuous time systems operating under a fast sampling rate $[9,10]$. The effects of quantization depend highly on the structure of the controller. This paper seeks to reduce these errors in the synthesis of q-Markov COVERs.

\section{Discrete q-Markov COVER}

Consider the asymptotically stable nominal discrete system

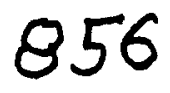




$$
\begin{aligned}
& x(k+1)=A x(k)+B u(k) ; x(k) \in R^{n_{z}}, u(k) \varepsilon \mathbb{R}^{n_{4}} \\
& y(k)=C x(k) \quad ; y(k) \in R^{n_{y}}
\end{aligned}
$$

where $\{\mathrm{u}(\mathrm{k})\}$ is a zero mean process with unit intensity $E\left\{\mathrm{u}(\mathrm{k}) \mathrm{u}^{*}(\mathrm{j})\right\}=\mathrm{I} \delta_{\mathrm{ij}}$ and $E\left\{\mathrm{x}(\mathrm{k}) \mathrm{u}^{*}(\mathrm{j})\right\}=0$ for $\mathrm{k} \geq \mathrm{j}$. The Markov parameters $\mathrm{M}_{\mathrm{i}}$ and covvariance parameters $\mathrm{R}_{\mathrm{j}}$ of (1.1) are defined by

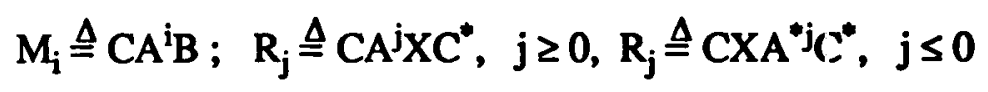

where the state covariance matrix $X$ satisfies the Lyapunov Equation

$$
\mathrm{X}=\mathrm{AXA}^{*}+\mathrm{BB}^{*} \text {. }
$$

These parameters $M_{i}$ and $R_{j}$ appear as coefficients in the expansion of the transfer function $\mathrm{H}(\mathrm{z})$ and power spectral density $\mathrm{H}(\mathrm{z}) \mathrm{H}^{*}\left(\mathrm{z}^{-1}\right)$; that is

$$
\mathrm{H}(\mathrm{z})=\mathrm{C}(\mathrm{zI}-\mathrm{A})^{-1} \mathrm{~B}=\sum_{\mathrm{i}=0}^{\infty} \mathrm{M}_{\mathrm{i}} \mathrm{z}^{-(\mathrm{i}+1)} ; \mathrm{H}(\mathrm{z}) \mathrm{H}^{*}(\mathrm{z})=\sum_{:=-\infty}^{\infty} \mathrm{R}_{\mathrm{j}} \mathrm{z}^{-\mathrm{j}}
$$

We suppose that as data we are given the first q-Markov and first q-covariance parameters $\left(\mathrm{M}_{\mathrm{i}}, \mathrm{R}_{\mathrm{i}} ; \mathrm{i}=0,1, \ldots, \mathrm{q}-1\right)$ of an asymptotically stable system from which we construct the two data matrices

$$
\begin{aligned}
& D_{\mathrm{q}} \triangleq R_{\mathrm{q}}-M_{\mathrm{q}} M_{\mathrm{q}}^{*} \varepsilon \mathrm{R}^{\mathrm{n}_{\mathrm{q}} \mathrm{q} \times \mathrm{m}_{\mathrm{q}} \mathrm{q}} \\
& \bar{D}_{\mathrm{q}} \triangleq R_{\mathrm{q}}-\bar{M}_{\mathrm{q}} \bar{M}_{\mathrm{q}}^{*} \varepsilon \mathrm{R}^{\mathrm{n}_{\mathrm{q}} \mathrm{q} \times \mathrm{m}_{\mathrm{q}} \mathrm{q}}
\end{aligned}
$$

where $R_{\mathrm{q}}, M_{\mathrm{q}}$ and $\bar{M}_{\mathrm{q}}$ are the Toeplitz matrices of the data as defined by

$$
\begin{gathered}
R_{\mathrm{q}} \triangleq\left[\begin{array}{llll}
\mathrm{R}_{0} & \mathrm{R}_{1}^{*} & \ldots & \mathrm{R}_{\mathrm{q}-1}^{*} \\
\mathrm{R}_{1} & \mathrm{R}_{0} & \ldots & \mathrm{R}_{\mathrm{q}-2}^{*} \\
\vdots & \vdots & & \vdots \\
\mathrm{R}_{\mathrm{q}-2} & \ldots & \ldots \\
\mathrm{R}_{\mathrm{q}-1} & \mathrm{R}_{\mathrm{q}-2} & \ldots & \mathrm{R}_{0}
\end{array}\right] \\
M_{\mathrm{q}} \triangleq\left[\begin{array}{ccccc}
0 & 0 & \ldots & 0 & 0 \\
\mathrm{M}_{0} & 0 & \ldots & 0 & 0 \\
\mathrm{M}_{1} & \mathrm{M}_{0} & \ldots & \vdots & . \\
\vdots & \vdots & & \vdots & \ldots \\
\mathrm{M}_{\mathrm{q}-2} & \mathrm{M}_{\mathrm{q}-3} & \ldots & \mathrm{M}_{0} & 0
\end{array}\right], \bar{M}_{\mathrm{q}} \triangleq\left[\begin{array}{cccc}
\mathrm{M}_{0} & 0 & \ldots & 0 \\
\mathrm{M}_{1} & \mathrm{M}_{3} & \ldots & 0 \\
\vdots & \vdots & \vdots & \vdots \\
\mathrm{M}_{\mathrm{q}-2} & & & 0 \\
\mathrm{M}_{\mathrm{q}-1} & \mathrm{M}_{\mathrm{q}-2} & \ldots & \mathrm{M}_{0}
\end{array}\right]
\end{gathered}
$$

The first data matrix $D_{\mathrm{q}}$ in (1.4a) is Hermitian and it is :hown in [3-4] to be 
positive semidefinite. Hence we can obtain a (nonunique) full rank factorization

$$
D_{q}=P_{q} P_{q}^{*} ; P_{q} \in R^{n_{q q} \times r_{q}} \text {, }
$$

where

$$
r_{q} \triangleq \operatorname{rank}\left(D_{q}\right)=\operatorname{rank}\left(P_{q}\right) \leq n_{y} q
$$

If we partition $\mathrm{P}_{\mathrm{q}}$ according to

$$
P_{q}^{*}=\left[E_{q}^{*} F_{q}^{*}\right] ; E_{q} \varepsilon R^{n_{y} x r_{q}}, F_{q} \in R^{(q-1) m_{y} \times r_{q}}
$$

then it follows that the second data matrix $\bar{D}_{\mathrm{q}}$ can be factored as

$$
\bar{D}_{\mathrm{q}}=\overline{\mathrm{P}}_{\mathrm{q}} \overline{\mathrm{P}}_{\mathrm{q}}^{*} ; \quad \overline{\mathrm{P}}_{\mathrm{q}} \in \mathrm{R}^{\mathrm{n}_{\mathrm{q}} \mathrm{q} \mathrm{r}_{\mathrm{q}}}
$$

where

$$
\bar{P}_{q}^{*}=\left[F_{q}^{*} G_{q}^{*}\right] ; G_{q} \in R^{n_{\gamma} x_{q}}
$$

for some $G_{q}$ (to be determined). The following result has been established.

\section{Theorem 1.1 [3]}

Given the $q$ Markov parameters $\left\{M_{i} ; i=0,1, \ldots, q-1\right\}$ and the $q$ covariance parameters $\left\{R_{i} ; i=0,1, \ldots, q-1\right\}$ and a matrix $G_{q}$ in (1.8) such that (1.7) is satisfied, then the realization $\left\{A_{q}, B_{q}, C_{q}\right\}$ of order $r_{q}$ defined by

$$
A_{q}=P_{q}^{+} \bar{P}_{q} ; B_{q}=P_{q}^{+}\left[M_{0}^{*} \cdots M_{q-1}^{*}\right]^{*} ; \quad C_{q}=E_{q}
$$

where $\mathrm{P}_{\mathrm{q}}^{+}$denotes the Moore-Penrose inverse of $\mathrm{P}$ is a $\mathrm{q}$-Markov COVER. The corresponding controllability grammian $X_{q}$ is given by

$$
X_{q}=I
$$

Furthermore

$$
P_{q}=\left[C_{q}^{*} A_{q}^{*} C_{q}^{*} \cdots\left(A_{q}^{q-1}\right)^{*} C_{q}^{*}\right]^{*}
$$

This theorem describes a large but not complete class $C_{\mathrm{q}}$ of q-Markov COVERs parameterized by $\left\{\mathrm{G}_{\mathrm{q}}\right\}$ such that for some $\mathrm{E}_{\mathrm{q}}, \mathrm{F}_{\mathrm{q}}$ the data matrices $D_{\mathrm{q}}, \bar{D}_{\mathrm{q}}$ satisfy (1.5)-(1.8). Each matrix $G_{q}$ will (generally) result in a $q-M a r k o v$ COVER having a different transfer function. In order to compute the set of all such $G_{q}$, observe in (1.5)-(1.8) that 


$$
D_{q}=\left[\begin{array}{c}
E_{q} \\
F_{q}
\end{array}\right]\left[E_{q}^{*} F_{q}^{*}\right] \text {. }
$$

Then

$$
\begin{gathered}
\bar{D}_{\mathrm{q}}=\left[\begin{array}{cc}
\bar{D}_{\mathrm{q}-1} & \overline{\mathrm{d}}_{\mathrm{q}} \\
\overline{\mathrm{d}}_{\mathrm{q}}^{*} & \overline{\mathrm{d}}_{\mathrm{qq}}
\end{array}\right]=\left[\begin{array}{c}
\mathrm{F}_{\mathrm{q}} \\
\mathrm{G}_{\mathrm{q}}
\end{array}\right]\left[\mathrm{F}_{\mathrm{q}}^{*} \mathrm{G}_{\mathrm{q}}^{*}\right] \\
\overline{\mathrm{d}}_{\mathrm{qq}} \in R^{n_{\gamma} \times \pi_{\mathrm{z}}}
\end{gathered}
$$

implies

$$
E_{q} E_{q}^{*}=R_{0}, F_{q} F_{q}^{*}=\bar{D}_{q-1}, F_{q} G_{q}^{*}=\bar{d}_{q}, G_{q} G_{q}^{*}=\bar{d}_{q q}
$$

Now expand $D_{q}$ in terms of its singular value decomposition

$$
D_{\mathrm{q}}=\left(U_{1} U_{2}\right)\left(\begin{array}{cc}
\Sigma_{1} & 0 \\
0 & 0
\end{array}\right)\left[\begin{array}{c}
U_{1}^{*} \\
U_{2}^{*}
\end{array}\right] ; \Sigma_{1} \in R^{\mathrm{q}_{4}}
$$

Then from (1.12a)

$$
\left(E_{q}^{*} F_{q}^{*}\right)=\Sigma_{1}^{1 / 2} U_{1}^{*}
$$

so that $E_{q}=C_{q}$ is defined by the first $n_{y}$ rows and $F_{q}$ by the last $(q-1) n_{y}$ rows of $\mathrm{U}_{1} \Sigma_{1}^{1 / 2}$. Define

$$
\rho_{q} \triangleq \operatorname{rank}\left(F_{q}\right)
$$

Then from (1.15)

$$
\rho_{q} \leq \min \left(r_{q},(q-1) n_{y}\right) \text {. }
$$

Next, expand $F_{q}$ in (1.13) in terms of its singular value decomposition. If strict inequality occurs in (1.16b) we have

$$
F_{q}=\left[U_{\alpha} U_{\beta}\right]\left[\begin{array}{cc}
\Sigma_{q} & 0 \\
0 & 0
\end{array}\right]\left[\begin{array}{c}
V_{\alpha}^{*} \\
V_{\beta}^{*}
\end{array}\right] ; \Sigma_{q} \in R^{P_{q} \times p_{q}}
$$

The Moore-Penrose inverse $\mathrm{F}_{\mathrm{q}}^{+}$of $\mathrm{F}_{\mathrm{q}}$ is then given by

$$
F_{q}^{+}=V_{\alpha} \Sigma_{q}^{-1} U_{\alpha}^{*}
$$

\section{Corollary 1.1}


Define
(i) $\mathrm{G}_{\mathrm{q} 1} \triangleq\left(\mathrm{F}_{\mathrm{q}}^{+} \overline{\mathrm{d}}_{\mathrm{q}}\right)^{*} \in R^{\mathrm{n}_{\mathrm{r}} \mathrm{x}_{\mathrm{q}}}$
(ii) $\mathrm{G}_{\mathrm{q} 2} \in R^{\mathrm{n}_{\mathrm{\gamma}} \mathrm{xs}}$ such that $\mathrm{G}_{\mathrm{q} 2} \mathrm{G}_{\mathrm{q} 2}^{*} \triangleq{\overline{\mathrm{d}_{\mathrm{qq}}}}-\overline{\mathrm{d}}_{\mathrm{q}}^{*} \overline{\mathrm{D}}_{\mathrm{q}-1}^{+} \overline{\mathrm{d}}_{\mathrm{q}}$

where

$$
s_{q} \triangleq \operatorname{rank}\left[\bar{d}_{q q}-\bar{d}_{q}^{*} \bar{D}_{q-1}^{+} \bar{d}_{q}\right]
$$

and

$$
\text { (iii) } \mathrm{G}_{\mathrm{q} 3} \triangleq \mathrm{V}_{\beta}^{*} \in R^{\left(\mathrm{r}_{\mathrm{q}}-\mathrm{p}_{\mathrm{q}}\right) \mathrm{xr}_{\mathrm{q}}}
$$

Then if strict inequality occurs in (1.16b) the set of all $G_{q}$ which satisfy (1.13) are given by

$$
G_{q}=G_{q 1}+G_{q 2} U_{q} G_{q 3}
$$

where

$$
U_{q} \in R^{s_{q} x\left(r_{q}-\rho_{q}\right)} ; s_{q} \leq r_{q}-\rho_{q} \leq n_{y}
$$

is an arbitrary row unitary matrix (i.e. $\mathrm{U}_{\mathrm{q}} \mathrm{U}_{\mathrm{q}}^{*}=\mathrm{I}$ ). Furthermore, if the MoorePenrose $\mathrm{P}_{\mathbf{q}}^{+}$of

$$
P_{q}=\left[E_{q}^{*} F_{q}^{*}\right]^{*}
$$

is expressed as

$$
\mathrm{P}_{\mathrm{q}}^{+}=\left[\begin{array}{ll}
\tilde{\mathrm{L}}_{11} & \mathrm{~L}_{12}
\end{array}\right] ; \tilde{\mathrm{L}}_{11} \in R^{\mathrm{r}_{\mathrm{q}} \times(\mathrm{q}-1)_{\mathrm{M}_{7}}}, \mathrm{~L}_{12} \in R^{\mathrm{r}_{\mathrm{q}} \mathrm{X}_{7}}
$$

then the corresponding state space representation $\left\{A_{q}, B_{q}, C_{q}\right\}$ of the $q-M a r k o v$ COVER is given by

$$
\begin{aligned}
& A_{q}=L_{11}+L_{12} G_{q} ; L_{11}=\tilde{L}_{11} F_{q} \in R^{I_{q} \mathrm{X}_{q}} \\
& B_{q}=P_{q}^{+}\left[M_{0}^{*} M_{1}^{*} \cdots M_{q-1}^{*}\right]^{*} ; C_{q}=E_{q} .
\end{aligned}
$$

If $r_{q}=\rho_{q}$, then $G_{q}=G_{q 1}$ is unique.

Proof: The expression for $F_{q} G_{q}^{*}$ in (1.13) implies $G_{q}^{*}$ is of the form

$$
\mathrm{G}_{\mathrm{q}}^{*}=\mathrm{F}_{\mathrm{q}}^{+} \overline{\mathrm{d}}_{\mathrm{q}}+\mathrm{G}_{\mathrm{q} 3}^{*} \mathrm{M}^{*} ; M \in R^{\mathrm{n}_{\mathrm{y}} \times\left(\mathrm{r}_{\mathrm{q}}-\mathrm{p}_{q}\right)}
$$

for some $M$. Then expanding $G_{q} G_{q}^{*}$ using (1.13) we have 


$$
\bar{d}_{q q}=\bar{d}_{q}^{*}\left(F_{q}^{+}\right)^{*} F_{q}^{+} \bar{d}_{q}+\bar{d}_{q}^{*}\left(F_{q}^{+}\right)^{*} G_{q 3}^{*} M^{*}+M G_{q 3} F_{q}^{+} \bar{d}_{q}-M G_{q 3} G_{q 3}^{*} M^{*}
$$

Also from (1.13) and (1.21)

$$
\left(F_{q}^{+}\right)^{*} F_{q}^{+}=\bar{D}_{q-1}^{+}, G_{q 3} G_{q 3}^{*}=I ;\left(F_{q}^{+}\right)^{*} G_{q 3}^{*}=0
$$

so that

$$
\mathrm{MM}^{*}=\overline{\mathrm{d}}_{\mathrm{qq}}-\overline{\mathrm{d}}_{\mathrm{q}}^{*}\left(\mathrm{~F}_{q}^{+}\right)^{*} \mathrm{~F}_{\mathrm{q}}^{+} \overline{\mathrm{d}}_{\mathrm{q}}
$$

Since $\mathrm{MM}^{*}$ has rank $\mathbf{s}_{\mathbf{q}}$,

$$
s_{q}=\operatorname{rank}\left(G_{q 2} G_{q}^{*}\right) \leq r_{q}-\rho_{q}
$$

\section{Optimal Finite Wordlength q-Markov COVER}

A fixed point finite wordlength realization of the ideal (i.e. infinite precision) q-Markov COVER (1.1) shall be referred to as a q-FWL Markov COVER and is described by

$$
\begin{gathered}
\hat{\mathbf{x}}(\mathbf{k}+1)=\hat{\mathrm{A}} \mathrm{Q}[\hat{\mathbf{x}}(\mathbf{k})]+\hat{\mathrm{B}} \hat{\mathrm{u}}(\mathrm{k}) \\
\hat{\mathrm{y}}(\mathbf{k})=\hat{\mathrm{C} Q}[\hat{\mathbf{x}}(\mathbf{k})] \\
\mathrm{Q}[\hat{\mathbf{x}}(\mathbf{k})]=\hat{\mathbf{x}}(\mathbf{k})-\mathrm{e}(\mathbf{k})
\end{gathered}
$$

where $e(k)$ is the error in computing $\hat{\mathbf{x}}(\mathbf{k})$. The components of the matrices $\hat{\mathrm{A}}, \hat{\mathbf{B}}$, $\hat{C}$ are assumed to have $a W_{0}$ bit fractional representation obtained by quantization of the components of $A, B, C$ in (1.1). The components of: $\hat{x}(k)$ have $a \mathrm{~W}+\mathrm{W}_{0}$ bit fractional part while components of $Q[\hat{x}(k)]$ and $\hat{u}(k)$ all have a $W$ bit fractional part. The components of the state residue vector $\mathrm{e}(\mathrm{k})$ has a $\mathrm{W}+\mathrm{W}_{0}$ bit fractional representation in which the most significant $W$ bits are zero. The LHS and RHS of (2.1) are therefore consistent with respect to their fractional wordlength representation. The number of bits required to represent the integer parts of $\hat{A}, \hat{B}$ and $\hat{C}$ depend on the dynamic range of the coefficients. Sitate space structures in which all coefficients are less than unity are therefore advantageous in this regard. The required integer representation of $Q[\hat{\mathbf{x}}(\mathbf{k})]$ will depend on the dynamic range of the input signal $\hat{\mathbf{u}}(\mathbf{k})$. Inadequate dynamic range will result in arithmetic overflow. The accuracy in the computation of $\hat{x}(\mathbf{k})$ is determined by its fractional wordlength $\mathbf{W}$.

Define the state error vector $\varepsilon_{\mathbf{x}}(k)$ and output error vector $\varepsilon_{y}(k)$ by 


$$
\varepsilon_{x}(k) \triangleq \hat{x}(k)-x(k) ; \varepsilon_{y}(k) \triangleq \hat{y}(k)-y(k)
$$

Then from (1.1), (2.1) and (2.2)

$$
\begin{aligned}
\varepsilon_{\mathrm{x}}(\mathrm{k}+1)= & \mathrm{A} \varepsilon_{\mathrm{x}}(\mathrm{k})-\mathrm{Ae}(\mathrm{k})+\Delta \mathrm{AQ}[\hat{\mathrm{x}}(\mathrm{k})]+\Delta \mathrm{Bu}(\mathrm{k})+\mathrm{B} \Delta \mathrm{u}(\mathrm{k}) \\
& \varepsilon_{\mathrm{y}}(\mathrm{k})=\mathrm{C} \varepsilon_{\mathrm{x}}(\mathrm{k})-\mathrm{Ce}(\mathrm{k})+\Delta \mathrm{CQ}[\hat{\mathrm{x}}(\mathrm{k})]
\end{aligned}
$$

where

$$
\begin{gathered}
\Delta A=\hat{A}-A ; \Delta B=\hat{B}-B ; \Delta C=\hat{C}-C \\
\Delta u(k)=\hat{u}(k)-u(k)
\end{gathered}
$$

There are five terms which contribute to the output error (i) internal arithmetic errors $e(k)$ due to state quantization (ii) coefficient errors due to errors $\Delta \mathbf{A}$ in $A$ (iii) $\Delta B$ in $B$ (iv) $\Delta C$ in $C$, and (v) input quantization errors $\Delta u(k)$. Under weak 'sufficiently exciting' conditions on the input $\{u(k)\}$ it can be shown [6] that if $\mathrm{Q}[\cdot]$ in (2.1) denotes 'roundoff' quantization, then $\{\mathrm{e}(\mathrm{k})\}$ is a zero mean uniform white process with covariance

$$
E\left\{\mathrm{e}(\mathrm{k}) \mathrm{e}^{*}(\mathrm{k})\right\}=\gamma^{2} \mathrm{I} ; \gamma^{2}=\frac{1}{12} 2^{-2 \mathrm{~W}} .
$$

Similarly $\{\Delta u(k)\}$ is assumed to be a zero mean white uniform process with

$$
E\left\{\Delta \mathrm{u}(\mathrm{k}) \Delta^{*} \mathrm{u}(\mathrm{k})\right\}=\gamma^{2} \mathrm{I}
$$

We assume that the quantized coefficients $\hat{A}, \hat{B}, \hat{C}$ are obtained by rounding $A, B$, $\mathrm{C}$ to $\mathrm{W}_{\mathrm{o}}$ bit fractions. Consequently, all components $\Delta \mathrm{p}$ of the error matrices $\Delta \mathrm{A}$, $\Delta B, \Delta C$ satisfy

$$
|\Delta \mathrm{p}|<\gamma_{0} ; \gamma_{0}=\frac{1}{2} 2^{-\mathrm{w}_{\bullet}} .
$$

For simplicity we normalize the error matrices and define $\delta \mathrm{A}, \delta \mathrm{B}, \delta \mathrm{C}$ by

$$
\delta \mathrm{A} \triangleq \frac{1}{\gamma_{0}} \Delta \mathrm{A} ; \delta \mathrm{B} \triangleq \frac{1}{\gamma_{0}} \Delta \mathrm{B} ; \delta \mathrm{C} \triangleq \frac{1}{\gamma_{0}} \Delta \mathrm{C}
$$

so that all components $\delta \mathrm{p}$ of $\delta \mathrm{A}, \delta \mathrm{B}$ and $\delta \mathrm{C}$ satisfy

$$
|\delta \mathrm{p}|<1 \text {. }
$$

The steady state output error covariance $Y$ of $\left\{\varepsilon_{y}(k)\right\}$ is then given by (we assume independence of $\varepsilon(k), e(k)$ and $\hat{x}(k))$. 


$$
\mathrm{Y}=\mathrm{CPC}+\gamma^{2} \mathrm{CC^{* }}+\gamma_{0}^{2}(\delta \mathrm{C})\left(\hat{\mathrm{X}}+\gamma^{2} \mathrm{I}\right)(\delta \mathrm{C})^{*}+\gamma_{0} \gamma^{2}\left[\mathrm{C}(\delta \mathrm{C})^{*}+(\delta \mathrm{C}) \mathrm{C}^{*}\right],
$$

where

$$
\begin{aligned}
\mathrm{P} & =E\left\{\varepsilon_{\mathrm{x}}(\mathrm{k}) \varepsilon_{\mathrm{x}}^{*}(\mathrm{k})\right\} \\
& =\mathrm{APA}^{*}+\gamma^{2} \mathrm{AA}^{*}+\gamma_{0}^{2}(\delta \mathrm{A})\left(\hat{\mathrm{X}}+\gamma^{2} \mathrm{I}\right)\left(\delta \mathrm{A}^{*}+\gamma_{0}^{2}(\delta \mathrm{B})(\delta \mathrm{B})^{*}+\gamma^{2} \mathrm{BB}^{*}\right.
\end{aligned}
$$

and

$$
\hat{\mathrm{X}}=E\left\{\hat{\mathrm{x}}(\mathrm{k}) \hat{\mathrm{x}}^{*}(\mathrm{k})\right\}=\hat{\mathrm{A}} \hat{\mathrm{X}}(\hat{\mathrm{A}})^{*}+\gamma^{2} \hat{\mathrm{A}}(\hat{\mathrm{A}})^{*}+\left(1+\gamma^{2}\right) \hat{\mathrm{B}} \hat{\mathrm{B}}^{*}
$$

For the remainder of this section we assume no coefficient errors (i.e. $\gamma_{0}=0$ in (2.9)) and consider only the effects due to finite state wordlength (FSWL). The issue of coefficient error shall be resumed in Section 4.

Theorem 2.1

Define the output noise measure

$$
\mathrm{J} \triangleq \mathrm{tr}[\mathrm{Y}]
$$

Then for $\gamma_{0}=0$

$$
J=\gamma^{2}\left[\operatorname{tr}[K]+\operatorname{tr}\left[B^{*} K B\right]\right\}
$$

where

$$
\mathrm{K}=\mathrm{A}^{*} \mathrm{KA}+\mathrm{C}^{*} \mathrm{C} \text {. }
$$

Proof: From (2.9)

$$
\mathrm{Y}=\mathrm{C} \overline{\mathrm{P}} \mathrm{C}^{*} ; \quad \overline{\mathrm{P}}=\mathrm{A} \overline{\mathrm{P}} \mathrm{A}^{*}+\boldsymbol{\gamma}^{2} \mathrm{Z}=\mathrm{P}+\boldsymbol{\gamma}^{2} \mathrm{I}
$$

where

$$
Z=I+B^{*}
$$

Now

$$
\overline{\mathrm{P}}=\gamma^{2} \sum_{k=0}^{\infty} A^{k} Z\left(A^{k}\right)^{*}
$$

and

$$
K=\sum_{k=0}^{\infty}\left(A^{k}\right)^{*} C^{*} C A^{k}
$$

so that 


$$
\operatorname{tr}\left[\overline{C P} C^{*}\right]=r^{2} t(Z K)
$$

A fixed point q-FSWL Markov COVER corresponding to the (ideal) qMarkov COVER (1.1) is therefore described by

$$
\begin{gathered}
\hat{\mathbf{x}}(\mathrm{k}+1)=\mathrm{AQ}[\hat{\mathbf{x}}(\mathbf{k})]+\mathrm{Bu}(\mathrm{k}) \\
\hat{\mathbf{y}}(\mathbf{k})=\mathrm{CQ}[\hat{\mathbf{x}}(\mathbf{k})] \\
\mathrm{Q}[\hat{\mathbf{x}}(\mathbf{k})]=\hat{\mathbf{x}}(\mathbf{k})-\mathrm{e}(\mathbf{k})
\end{gathered}
$$

The output noise gain $\left(\eta_{\mathrm{x}}\right)$ due to state quantization and the output noise gain $\left(\eta_{u}\right)$ due to input quantization are defined by

$$
\left.\eta_{\mathrm{x}} \triangleq \mathrm{tr}[\mathrm{K}] ; \eta_{\mathrm{u}} \triangleq \mathrm{tr}_{\mathrm{B}}{ }^{*} \mathrm{~KB}\right]
$$

The noise gain $\eta_{x}$ generally varies with state space representation whereas $\eta_{u}$ is independent of the coordinate basis. Specifically, consider the q-FSWL Markov COVER

$$
\begin{gathered}
\hat{\mathrm{z}}(\mathrm{k}+1)=A \mathrm{Q}[\hat{\mathrm{z}}(\mathrm{k})]+B \hat{\mathrm{u}}(\mathrm{k}) \\
\mathrm{y}(\mathrm{k})=C \mathrm{Q}[\hat{\mathrm{z}}(\mathrm{k})] \\
\mathrm{Q}[\hat{\mathrm{z}}(\mathrm{k})]=\hat{\mathrm{z}}(\mathrm{k})-\mathrm{f}(\mathrm{k})
\end{gathered}
$$

where

$$
A=\mathrm{T}^{-1} \mathrm{AT}, B=\mathrm{T}^{-1} \mathrm{~B}, C=\mathrm{CT}
$$

and $Q[\hat{z}(k)]$ has a W bit fractional representation. Assuming 'sufficient excitation' by $\hat{u}(k)$, the state residue sequence $\{f(k)\}$ in $(2.14 a)$ due to roundoff quantization will again be a zero mean white uniform process with covariance $\gamma^{2} I$ as in (2.5). The corresponding output quantization noise gains $\eta_{z}$ and $\tilde{\eta}_{u}$ due respectively to state and input quantization are given by

$$
\eta_{\mathrm{z}}=\operatorname{tr}\left[\mathrm{K}_{\mathrm{z}}\right] ; \tilde{\eta}_{\mathrm{u}}=\operatorname{tr}\left[B^{*} \mathrm{~K}_{\mathrm{z}} B\right]
$$

where $B$ is given by $(2.14 \mathrm{~b})$ and

$$
\mathrm{K}_{\mathrm{z}}=A \mathrm{~K}_{\mathrm{z}} A^{*}+C^{*} C \text {. }
$$

But from (2.11), $\mathrm{K}_{\mathrm{z}}=\mathrm{T}^{*} \mathrm{KT}$, so that 


$$
\eta_{z}=\operatorname{tr}\left[T^{*} K T\right] ; \tilde{\eta}_{u}=\operatorname{tr}\left[B^{*} K B\right]
$$

Notice from (2.13) that the noise gain $\eta_{u}$ due to input quantization errors is unaffected by a similarity transformation. Conversely the noise gain $\eta_{\mathrm{x}}$ due to state quantization generally changes with co-ordinate bases. There is no change if $T$ is unitary. The q-FSWL Markov COVER (2.14) is superior to the q-FSWL Markov COVER (2.12) if

$$
\eta_{\mathbf{z}}<\eta_{\mathbf{x}}
$$

However the comparison in (2.18) must be made under the assumption of identical scaling of the states $\hat{x}(k)$ and $\hat{z}(k)$. Specifically, equal $\mathrm{l}_{2}$-scaling of gain $\alpha$ from a zero mean unit intensity white noise input $\hat{u}(k)$ to the state components $\hat{\mathbf{x}}_{\mathrm{j}}(\mathbf{k})$ of $\hat{\mathbf{x}}(\mathbf{k})$ requires

$$
\mathrm{X}_{\mathrm{jj}}=\alpha \text { for all } \mathrm{j}
$$

where $X_{\mathrm{ij}}$ denotes the $\mathrm{jth}$ diagonal component of the state covariance matrix $\mathbf{X}$ given by (1.3). Equal $l_{2}$-scaling of gain $\alpha$ of components of $\hat{z}(k)$ in (2.14) requires

$$
Z_{\mathrm{jj}}=\alpha ; Z=A Z A^{*}+B B^{*}
$$

Equality in $\mathrm{l}_{2}$-scaling of representations (2.12) and (2.14) is equivalent to equality in the state dynamic range (i.e. number of bits in the integer representation of states) for a given probability of overflow. We now state a result which is important for establishing $\mathrm{l}_{2}$-scaling.

Lemma 2.1 $[8,9]$ Suppose $M=M^{*}>0$ is an nxn matrix. Then a necessary and sufficient condition for the existence of a unitary matrix $V$ such that

$$
\mathrm{VMV}_{\mathrm{ij}}^{*}=\alpha \text { for all } \mathrm{j}
$$

is

$$
\operatorname{tr}[\mathrm{M}]=\mathrm{n} \alpha
$$

We have shown in Lemma 1.1 that different similarity trantsformations of an ideal q-Markov COVER corresponds to different factorization of the first data matrix $D_{\mathrm{q}}$ in (1.5a). Our aim is to optimize this factorization.

\section{Definition 2.1}


The Optimal q-FSWL Markov COVER minimizes the output quantization noise gain $\eta$ due to state quantization errors; that is

$$
\eta_{\text {opt }}=\min _{T, G_{q}} t\left[T^{*} K_{q} T\right] ; T^{*} T=\Lambda^{-1}
$$

subject to the $\mathrm{l}_{2}$-scaling constraint:

$$
\Lambda_{\mathrm{ij}}=\alpha \text { for all } \mathrm{j}
$$

where the observability grammian $\mathrm{K}_{\mathrm{q}}$ satisfies

$$
\mathrm{K}_{\mathrm{q}}=\mathrm{A}_{\mathrm{q}}^{*} \mathrm{~K}_{\mathrm{q}} \mathrm{A}_{\mathrm{q}}+\mathrm{C}_{\mathrm{q}}^{*} \mathrm{C}_{\mathrm{q}}
$$

with $\left\{A_{q}, B_{q}, C_{q}\right\}$ defined by (1.22)-(1.25).

In corollary 1.1 we have shown that all the degrees of freedom available to select $G_{q}$ are confined to an arbitrary row unitary matrix $U_{q}$. We now show how to optimize $U_{\mathbf{q}}$.

\section{Theorem 2.1}

a. The optimal q-FSWL Markov COVER (1.25) is defined by

$$
\eta_{\text {opt }}=r_{q}^{-1} \min _{U_{q}}\left(t r\left[K_{q}^{1 / 2}\right]\right)^{2}
$$

where $U_{q} \in R^{\delta_{q} x\left(r_{q}-P_{q}\right)}$ is an arbitrary row unitary matrix and $\mathrm{K}_{q}$ satisfies (2.23).

b. The transfer function of the optimal q-FSWL Markov COVER has Hankel singular values given by the eigenvalues of $K_{q}$ defined by the minimizing $\mathbf{U}_{\mathbf{q}}$.

c. Suppose $\mathrm{U}_{\mathrm{q}}=\mathrm{U}_{\mathrm{qo}}$ is the minimizing solution corresponding to the optimal $\mathrm{G}_{\mathrm{q}}=\mathrm{G}_{\mathrm{qo}}$ in (1.22a). Let $\left(\mathrm{A}_{\mathrm{qo}}, \mathrm{B}_{\mathrm{qo}}, \mathrm{C}_{\mathrm{qo}}\right\}$ be the corresponding state space realization in (1.24). Then the optimal q-FSWL Markov COVER has a (nonunique) state space representation $\left[\mathrm{T}_{\mathrm{o}}^{-1} \mathrm{~A}_{\mathrm{qo}} \mathrm{T}_{\mathrm{o}}, \mathrm{T}_{\mathrm{o}}^{-1} \mathrm{~B}_{\mathrm{qo}}, \mathrm{C}_{\mathrm{qo}} \mathrm{T}\right\}$ where

$$
T_{0}=U_{0} \pi_{0} V_{0}^{*}
$$

such that

(i) the unitary matrix $U_{o}$ is defined by 


$$
\mathrm{U}_{0}^{*} \mathrm{~K}_{q_{0}} \mathrm{U}_{\mathrm{o}}=\Sigma_{0}^{2}
$$

where

$$
K_{q o}=A_{q o} K_{q o} A_{q 0}^{*}+C_{q o}^{*} C_{q o} ; \Sigma_{0}^{2}=\operatorname{diag}\left\{\sigma_{10}^{2}, \sigma_{i 20}^{2} \ldots, \sigma_{r_{p} \rho}^{2}\right\}
$$

in which $\left\{\sigma_{j 0}^{2}\right\}$ are the optimal Hankel singular values (eigenvalues of $K_{q_{0}}$ ).

(ii)

$$
\pi_{0}^{2}=\frac{1}{\alpha^{2} r_{q}}\left(\sum_{k=1}^{r_{q}} \sigma_{k 0}\right) \Sigma_{0}^{-1}
$$

and (iii) $V_{0}$ is unitary such that

$$
\begin{gathered}
\left(V_{0} \Sigma_{0} V_{o}^{*}\right)_{j j}=\frac{\sum_{k=1}^{r_{q}} \sigma_{k o}}{r_{q}} \text { for all j } \\
\eta_{\text {opt }} \triangleq \eta_{q} \text { (optimal) }=\frac{1}{\alpha^{2} r_{q}}\left(\sum_{k=1}^{r_{q}} \sigma_{k o}\right)^{2}
\end{gathered}
$$

Proof: By corollary 1.1 we have for $G_{q}$ defined by (1.2\%) for any row unitary matrix $\mathrm{U}_{\mathrm{q}}$ (of appropriately specified dimensions) that $\mathrm{C}_{\mathrm{q}}$ defines a $\mathrm{q}$-Markov COVER. The corresponding realization $\left\{A_{q}, B_{q}, C_{q}\right\}$ for earh such $U_{q}$ has identity controllability grammian and observability grammian $K_{q}$ defined by (2.23). Now given a particular $\mathrm{U}_{\mathbf{q}}$, apply a similarity transformation

$$
\mathrm{T}=\mathrm{U}_{\mathrm{o}} \boldsymbol{\pi}_{\mathbf{0}} \mathrm{V}_{\mathrm{o}}^{*}
$$

to the given q-Markov COVER. Then

$$
\operatorname{tr}\left(T^{*} K_{q} T\right)=\operatorname{tr}\left(\pi_{0}^{2} U_{0}^{*} K_{q} U_{0}\right)
$$

and

$$
\left(\mathrm{T}^{*} \mathrm{~T}^{-1}=\mathrm{V}_{0} \pi_{0}^{-2} \mathrm{~V}_{0}^{*}\right.
$$

By lemma 2.1, the $1_{2}$-scaling constant can be satisfied for some $V_{0}$ provided $\operatorname{tr}\left(\pi_{0}^{-2}\right)=n \alpha$. Following Williamson [1, Theorem 4.1] (with a minor modification of the $\mathrm{l}_{2}$-scaling constraint), the optimal performance is given by 


$$
\eta_{\text {qopt }}=\frac{\left(\sum_{\alpha=1}^{T_{q}} \sigma_{j}\right)^{2}}{\alpha^{2} r_{q}}
$$

where $\left\{\sigma_{j}^{2}\right\}$ are the eigenvalues of $K_{q}$. That is,

$$
\operatorname{tr}\left(\mathrm{K}_{q}^{1 / 2}\right)=\sum_{j=1}^{\mathrm{r}_{4}} \sigma_{j}
$$

The optimal q-FSWL Markov COVER therefore achieves the minimum in (2.24). The structure of $U_{0}, \pi_{0}, V_{0}$ in (2.25)-(2.29) follow directly from Williamson [1] (see proof of Theorem 4.1 with $U=$ I).

\section{Computation of the Optimal FSWL Markov COVER}

Necessary conditions for the optimal solution in Theorem 2.1 can be obtained using the method of Lagrange multipliers. Specifically, let

$$
J=\left(\operatorname{tr}\left[K_{q}^{1 / 2}\right]\right)^{2}+\operatorname{tr}\left[\Lambda\left(-K_{q}+A_{q}^{*} K_{q} A_{q}+C_{q}^{*} C_{q}\right)\right]+\operatorname{tr}\left[\Omega\left(I-U_{q} U_{q}^{*}\right)\right]
$$

where

$$
\mathrm{K}_{\mathrm{q}}=\mathrm{K}_{\mathrm{q}}^{3 / 2} \mathrm{~K}_{1}^{1 / 2^{*}} \Lambda=\Lambda^{*} \in R^{\mathrm{T}_{\mathrm{q}} \mathrm{xT}_{\mathrm{q}}} ; \Omega=\Omega^{*} \in R^{\mathrm{s}_{\mathrm{q}} \mathrm{Xs}_{\mathrm{q}}}
$$

are symmetric Lagrange multipliers. After taking derivatives of $\mathrm{J}$ using (1.22) and (1.25)

$$
\begin{gathered}
\frac{\partial J}{\partial \Lambda}=-K_{q}+A_{q}^{*} K_{q} A_{q}+C_{q}^{*} C_{q} \\
\frac{\partial J}{\partial \Omega}=I-U_{q} U_{q}^{*} \\
\frac{\partial J}{\partial K_{q}^{3 / 2}}=2 I-2 \Lambda K_{q}^{3 / 2}+2 A_{q} \Lambda A_{q}^{*} K_{q}^{1 / 2} \\
\frac{\partial J}{\partial U_{q}}=2 G_{q 2}^{*} L_{12}^{*} K_{q} A_{q} \Lambda G_{q 3}^{*}-2 \Omega U_{q}
\end{gathered}
$$

By setting these derivatives to zero we obtain the following result.

Lemma 3.1 Necessary conditions for the derivation of the optimal q-FSWL Markov COVER are given by 


$$
\begin{aligned}
& K_{q}=A_{q}^{*} K_{q} A_{q}+C_{q}^{*} C_{q} \\
& \Lambda=A_{q} \Lambda A_{q}^{*}+K_{q}^{-1 / 2} ; \quad \Lambda=\Lambda^{*} \in R^{\mathrm{r}_{q} X x_{q}} \\
& \mathrm{U}_{\mathrm{q}} \mathrm{U}_{\mathrm{q}}^{*}=\mathrm{I} \quad ; \mathrm{U}_{\mathrm{q}} \in R^{\mathrm{s}_{\mathrm{q}} \mathrm{x}\left(\mathrm{r}_{\mathrm{q}}-P_{\mathrm{q}}\right)} \\
& \Omega U_{q}-P_{q} U_{q} Q_{q}=R_{q} ; \Omega=\Omega^{*} \in R^{s_{q} \times s_{q}}
\end{aligned}
$$

where

$$
\begin{gathered}
P_{q}=P_{q}^{*}=G_{q 2}^{*} L_{12}^{*} K_{q} L_{12} G_{q 2} \in R^{s_{q} \times s_{q}} \\
Q_{q}=Q_{q}^{*}=G_{q 3} \Lambda G_{q 3}^{*} \in R^{\left(r_{q}-p_{q}\right) \times\left(r_{q}-P_{q}\right)} \\
R_{q}=G_{q 2}^{*} L_{12}^{*} K_{q}\left(L_{11}+L_{12} G_{q 1}\right) \Lambda G_{q 3}^{*} \in R^{s_{q} \times\left(I_{q}-P_{q}\right)}
\end{gathered}
$$

and $A_{q}, G_{q j}, L_{i j}$ are defined by (1.20)-(1.24)

These necessary conditions cannot be solved explicitly for the optimal row unitary matrix $U_{\mathbf{q}}$ and so an iterative procedure is required. One possible algorithm is now described.

Recursive Algorithm for Optimal q-FSWL Markov COVER:

(0) Set $\mathrm{j}=0$ and choose any row unitary $\mathrm{U}_{\mathrm{q}}(0)$ in (1.21a)

(1) Form $A_{q}(j)$ from

$$
A_{q}(j)=\left(L_{11}+L_{12} G_{q 1}\right)+L_{12} G_{q 2} U_{q}(j) G_{q 3}
$$

(2) Compute $K_{q}(j): K_{1}(j)=A_{q}^{*}(j) K_{q}(j) A_{q}(j)+C_{q}^{*} C_{q}$

(3) Compute $\Lambda(j): \Lambda(j)=A_{q}(j) \Lambda(j) A_{q}^{*}(j)+K_{q}^{-3 / 2}(j) ; \Lambda(j)=\Lambda^{*}(j)(3.5 c)$

(4) Compute $P_{q}(j), Q_{q}(j) R_{q}(j)$ :

$$
\begin{gathered}
P_{q}(j)=G_{q 2}^{*} L_{12}^{*} K_{q}(j) L_{12} G_{q 2} ; Q_{q}(j)=G_{q 3} \Lambda(j) G_{q 3}^{*} ; \\
R_{q}(j)=G_{q 2}^{*} L_{12}^{*} K_{q}(j)\left(L_{11}+L_{12} G_{q 1}\right) \Lambda(j) G_{q 3}^{*}
\end{gathered}
$$


(5) Update $U_{q}(j)$ by solving the nonlinear algebra problem:

$$
\begin{gathered}
\Omega(j) U_{q}(j+1)-P_{q}(j) U_{q}(j+1) Q_{q}(j)=R_{q}(j) ; \quad \Omega(j)=\Omega^{*}(j) \\
U_{q}(j+1) U_{q}^{*}(j+1)=I
\end{gathered}
$$

The most difficult step at each stage of the algorithm is to solve (3.5e) for a row unitary $U_{q}(j+1)$ and symmetric $\Omega(j)$. There is generally no explicit solution except for the following special cases.

Lemma 3.2 Consider the equation

$$
\Omega U_{q}-P_{q} U_{q} Q_{q}=R_{q} ; \Omega \in R^{s_{q} R_{q}}
$$

where

$$
P_{q}=P_{q}^{*} \in R^{s_{q} \times x_{4}} ; Q_{q}=Q_{q}^{*} \in R^{\left(x_{q}-P_{q}\right)\left(x_{q}-P_{q}\right)} ; R_{q} \in R^{s_{q} x\left(x_{q}-P_{q}\right)}
$$

are given. Then there exists an analytical solution $\left(\Omega, U_{q}\right)$ with $\Omega$ symmetric and $U_{q}$ row unitary when $\mathrm{s}_{\mathrm{q}}=1$ or $\mathrm{Q}_{\mathrm{q}}=\beta I$. ( $\beta$ scalar)

a. When $s_{q}=1, \Omega$ and $P_{q}$ are scalars and $R_{q}$ is a row vector. Then $U_{q}$ is arbitrary for $R_{q}=0$ while for $R_{q} \neq 0$

$$
U_{q}=R_{q}\left(\Omega I-P_{q} Q_{q}\right)^{-1} ;\left\|U_{q}\right\|=1
$$

b. When $\mathrm{Q}_{q}=\beta I$, let $\mathrm{R}_{q} \mathrm{R}_{q}^{*}$ have the singular value decomposition

$$
R_{q} R_{q}^{*}=\left(V_{1} V_{2}\right)\left(\begin{array}{cc}
\Sigma_{q 1} & 0 \\
0 & 0
\end{array}\right]\left[\begin{array}{l}
V_{1}^{*} \\
V_{2}^{*}
\end{array}\right]
$$

where $\Sigma_{q 1}$ is invertible. Then

$$
U_{q}=\left(V_{1}^{*}\right)^{+} \Sigma_{q 1}^{-1 / 2} V_{1}^{*} R_{q} ; \Omega=\beta P_{q}+V_{1} \Sigma_{q 1}^{1 / 2} V_{1}^{*}
$$

In particular, when $R_{q} R_{q}^{*}$ has full rank,

$$
U_{q}=\left(R_{q} R_{q}^{*}\right)^{-1 / 2} R_{q}
$$

Proof: For case (a)

$$
\mathrm{U}_{\mathrm{q}}\left(\Omega I-\mathrm{P}_{\mathrm{q}} \mathrm{Q}_{\mathrm{q}}\right)=\mathrm{R}_{\mathrm{q}} ; \quad \Omega \text { scalar }
$$

so that (3.8) follows by the row unitary property $U_{q} U_{q}^{*}=I$. In case (b) 


$$
\left(\Omega-\beta P_{q}\right) U_{q}=R_{q}
$$

and using the row unitary property of $\mathrm{U}_{\mathrm{q}}$

$$
\left(\Omega-\beta P_{q}\right)^{2}=R_{q} R_{q}^{*}
$$

Hence using the SVD of $R_{q} R_{q}^{*}$

$$
\mathrm{V}_{1} \sum_{\mathrm{q}}^{1 / 2} \mathrm{~V}_{1}^{*} \mathrm{U}_{\mathrm{q}}=\mathrm{R}_{\mathrm{q}}
$$

But $V_{1}^{*} V_{1}=I$ and $V_{1}^{*}$ has full row rank which gives (3.9).

Strictly speaking, (3.8) is not an analytical solution since the scalar $\Omega$ must still be chosen so that $\left\|U_{q}\right\|=1$. Note that by Corollary $1.1, G_{q_{3}} G_{q_{3}}^{*}=I$ so that $Q_{q}(j)=I$ in $(3.5 b)$ if $\Lambda(j)=I$. The necessary condition (3.5e) is equivalent to assuming $K_{q}(j), \Lambda(j), P_{q}(j), Q_{q}(j)$ and $R_{q}(j)$ are known and optimizing over row unitary $U_{q}(j+1)$. That is, after dropping the index $j$ and $j+1$ in (3.5e) we have the following result.

Lemma 3.3 Suppose $P_{q}, Q_{q}$ and $R_{q}$ in (3.7) are known. Then a necessary condition for a row unitary matrix $U_{q}$ to achieve optimally for the problem:

$$
\min _{\mathrm{U}_{\mathrm{q}}} \operatorname{tr}\left[\mathrm{Q}_{\mathrm{q}} \mathrm{U}_{\mathrm{q}}^{*} \mathrm{P}_{\mathrm{q}} \mathrm{U}_{\mathrm{q}}+2 \mathrm{R}_{\mathrm{q}} \mathrm{U}_{\mathrm{q}}\right] ; \mathrm{U}_{\mathrm{q}} \in R^{s_{\mathrm{q}} \mathrm{x}\left(\mathrm{I}_{\mathrm{q}}-\mathrm{P}_{\mathrm{q}}\right)}
$$

is that there exists a symmetric matrix $\Omega$ such that (3.6) is satisfied.

Furthermore, the optimization in (3.11) is equivalent to

$$
\min _{\mathrm{U}} \mathrm{J}(\mathrm{U}) ; \mathrm{U} \in R^{\left(\mathrm{x}_{\mathrm{q}}-P_{q}\right) \times\left(\mathrm{r}_{q}-\mathrm{P}_{q}\right)}
$$

where

$$
\mathrm{J}(\mathrm{U})=\mathrm{tr}[\mathrm{QU} * \mathrm{PU}+2 \mathrm{RU}]
$$

over unitary matrices $U^{*}=\left[U_{q}^{*} V_{q}^{*}\right]$ where $Q=Q_{q}$ and

$$
\begin{gathered}
P=P^{*}=\left[\begin{array}{cc}
P_{q} & 0 \\
0 & 0
\end{array}\right] \in R^{\left(x_{q}-P_{q}\right)\left(x_{q}-P_{q}\right)} \\
R=\left[R_{q} 0\right] \in R^{\left(r_{q}-P_{q}\right) x\left(r_{q}-P_{q}\right)}
\end{gathered}
$$

The advantage of the point of view (3.12) is that $U$ can be treated as a square

$$
871
$$


matrix. The solution to (3.12) when $U$ is a $2 \times 2$ unitary matrix is provided in the following lemma. The result can be derived by directly substituting into (3.12).

Lemma 3.4 Suppose $P=P^{*}=\left[p_{i j}\right], Q=Q^{*}=\left[q_{i j}\right]$ and $R=\left[r_{i j}\right]$ are $2 \times 2$ matrices. Then the minimum in (3.12) over $2 \times 2$ unitary matrices $U$ is achieved by either

(i) $\quad U=\operatorname{diag}\left\{u_{1}, u_{2}\right\}$ where $u_{1}^{2}=1, u_{2}^{2}=1$ minimize

$$
J_{1}=r_{11} u_{1}+r_{22} u_{2}+2 q_{12} p_{12} u_{1} u_{2}
$$

or (ii)

$$
U=\left[\begin{array}{cc}
x & \sqrt{1-x^{2}} \\
-\sqrt{1-x^{2}} & x
\end{array}\right]
$$

where $|\mathbf{x}| \leq 1$ minimizes

$$
\begin{gathered}
J_{2}(x)=a x^{2}+2 b x+2(c x+d) \sqrt{1-x^{2}} \\
a=\left(p_{11}-p_{22}\right)\left(q_{11}-q_{22}\right), b=r_{11}+r_{22} \\
c=q_{12}\left(p_{11}-p_{22}\right)+p_{12}\left(q_{22}-q_{11}\right), d=r_{21}-r_{12}
\end{gathered}
$$

Note that we must optimize over the disjoint sets of $2 \times 2$ unitary matrices consisting of signature matrices (as in (3.13)) and rotations (as in (3.14)). The optimal solution of (3.13) can be obtained by inspection of the magnitudes of the coefficients in $u_{j}$. For example, suppose

$$
\left|r_{11}\right| \geq\left|q_{12} p_{12}\right| \geq\left|r_{22}\right|
$$

Then

$$
u_{1}=-\operatorname{sgn}\left(r_{11}\right) ; u_{1} u_{2}=-\operatorname{sgn}\left(q_{12} p_{12}\right)
$$

However the optimization in (3.14) requires numerical solution.

A general nxn unitary matrix $U$ is either a signature matrix (i.e. a diagonal matrix $\Sigma$ such that $\Sigma^{2}=I$ ) or a product of $1 / 2 n(n-1)$ rotations $U_{i j}$ where the components of $U_{i j}(k, l) U_{i j}$ are defined by

$$
U_{i j}(i, i)=U_{i j}(j, j)=\cos \theta_{i j}
$$




$$
\begin{gathered}
U_{i j}(i, j)=-U_{i j}(j, i)=\sin \theta_{i j} \\
U_{i j}(k, k)=1 \text { for } k \neq i, k \neq j \\
U_{i j}(k, l)=0 \text { otherwise }
\end{gathered}
$$

A particular signature matrix is also defined by $(3.15 \mathrm{~b})$ where

$$
\begin{aligned}
& U_{i j}(k, k)= \pm 1 \text { for } k=i, j \\
& U_{i j}(k, 1)=0 \text { for } k \neq 1
\end{aligned}
$$

By letting

$$
\mathrm{U}=\prod_{\mathbf{i} \mathbf{j}} \mathrm{U}_{\mathbf{i j}}
$$

The optimization in (3.12) can be reduced to a sequence of one dimensional optimizations over the angles $\theta_{\mathrm{ij}}$. To be complete, $\mathrm{J}(\mathrm{U})$ should also be evaluated separately for all $2^{n}\left(n=r_{q}-\rho_{q}\right)$ signature matrices. A compromise during the iterative procedure is to include the possibility of components $U_{i j}$ being defined by (3.16) as well as (3.15a). Rather than present the general result we only illustrate by means of an example.

Specifically, suppose we express a $3 \times 3$ unitary matrix $U$ as

$$
\mathrm{U}=\mathrm{U}_{12} \mathrm{U}_{13} \mathrm{U}_{23}
$$

Then by invoking the trace property, $\mathrm{J}$ in (3.12b) can equivalently be expressed as

$$
J\left(U_{i j}\right)=\operatorname{tr}\left[Q_{i j} U_{i j}^{*} P_{i j} U_{i j}+2 R_{i j} U_{i j}\right]
$$

where

$$
\begin{aligned}
& Q_{12}=U_{12} U_{23} Q_{23}^{*} U_{13}^{*} ; P_{12}=P ; R_{12}=U_{23} U_{13} R \\
& Q_{13}=U_{23} Q_{23}^{*} ; P_{13}=U_{12}^{*} P U_{12} ; R_{13}=U_{23} R U_{12} \\
& Q_{23}=Q ; \quad P_{23}=U_{13}^{*} U_{12}^{*} P_{12} U_{13} ; R_{23}=R_{12} U_{13}
\end{aligned}
$$

With $i=i_{o}$, and $j=j_{o}$ fixed in (3.18a), $J$ can be optimization over $U_{i_{j} j_{0}}$. The procedure is recursive. That is, first assume $i=1, j=2$ with $U_{13}$ and $U_{23}$ both initialized to (say) the identity. After optimizing over $U_{12}$, fix $U_{12}$ and $U_{13}$ and optimize over $U_{23}$, etc. Many cycles may be necessary for convergence.

In order to explicitly demonstrate the formulation for each of the $2 \times 2$ optimizations consider the case $i=1, j=2$, and express 


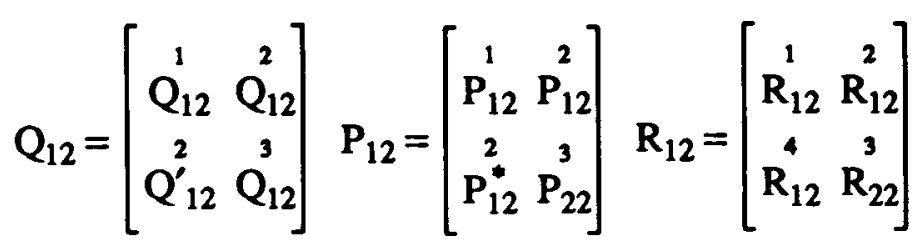

where $Q_{12}^{1}, P_{12}^{1}, R_{12}^{1} \in R^{2 \times 2}$. Then from (3.15), (3.16) the optimal $\theta_{12}$ which minimizes $\mathrm{J}_{12}\left(\mathrm{U}_{12}\right)$ also minimizes

$$
\tilde{\mathrm{J}}_{12}\left(\theta_{12}\right)=\operatorname{tr}\left[\mathrm{Q}_{12}^{1} \mathrm{U}_{\theta}^{*} \mathrm{P}_{12}^{1} \mathrm{U}_{\theta}+2\left(\mathrm{R}_{11}+\mathrm{Q}_{12}^{2} \mathrm{P}_{12}^{2}\right) \mathrm{U}_{\theta}\right]
$$

where components of the $2 \times 2$ unitary matrix $U_{\theta}^{*}$ is defined by (3.15a) or (3.16) for $i, j, \in\{1,2\}$ The $2 \times 2$ optimization of $\tilde{J}_{12}\left(\theta_{12}\right)$ over $\theta_{12}$ is partially solved in lemma 3.4.

Before concluding this section it is important to reiterate that the dimension of the problem for optimizing over the row unitary matrices $U_{q}$ is generally low. In particular from (1.21b) both the number of rows and columns of $U_{q}$ is not greater than the number of outputs. For a single output systems, $U_{q}$ is a scalar and so there are at most two possibilities, and no optimization is necessary. That is, for $\rho_{q}<r_{q}$ we merely evaluate the cost in (2.24) for two values of $G_{q}$ in (1.21a) corresponding to $U_{q}= \pm 1$, while if $\rho_{q}=r_{q}$, then $G_{q}=G_{q 1}$ is unique.

\section{Coefficient Errors}

Recall that $Y$ in (2.9) is the error in the covariance of the output $\{\hat{y}(k)\}$ due to finite precision implementation of both states and coefficients of the q-Markov COVER. The optimal q-FSWL Markov COVER minimizes the trace of $Y$ when there are no coefficient errors (corresponding to $\gamma_{0}=0$ ). Furthermore, when there are no coefficient errors, there are no errors in either the Markov parameters $\mathbf{M}_{\mathbf{i}}$ or covariance parameters $R_{j}$ in (1.2). Once coefficient errors are introduced and all finite wordlength (FWL) errors are considered, there is no longer a clear interpretation of what should constitute the optimal q-FSWL Markov COVER. One possibility is to again attempt to minimize the trace of $Y$. Alternative performance criteria could be based on the errors $\Delta \mathrm{M}_{\mathrm{i}}$ and $\Delta \mathrm{R}_{\mathrm{j}}$ in the Markov and covariance parameters as given by

$$
\begin{aligned}
& M_{i}+\Delta M_{i}=(C+\Delta C)(A+\Delta A)^{i}(B+\Delta B) \\
& R+\Delta R_{j}=(C+\Delta C)(A+\Delta A)^{j} \bar{X}(C+\Delta C)^{*}
\end{aligned}
$$

where $\bar{X}$ satisfies $\bar{X}=A \bar{X} A^{*}+B B^{*}$. For example, one could attempt to minimize 


$$
\left.C_{\mathrm{M}} \triangleq \sum_{\mathrm{i}=0}^{\mathrm{q}} \mathrm{tr}\left[\Delta \mathrm{M}_{\mathrm{i}}\left(\Delta \mathrm{M}_{\mathrm{i}}\right)^{*}\right] \text { or } C_{\mathrm{R}} \triangleq \sum_{\mathrm{i}=0}^{\mathrm{q}} \mathrm{tr} \mid \Delta \mathrm{R}_{\mathrm{i}}\right]
$$

However there are no results which directly connect $C_{\mathrm{M}}$ or $C_{\mathrm{R}}$ with errors in time or frequency response of the q-Markov COVERs. Fur:hermore, the analytical and computational aspects involved in the resulting optimization would be very difficult if not practically impossible.

A convenient approach to parameter optimization is to assume a statistical model for parameter errors. A statistical design can be justified along the following lines. Suppose (as is the case in practice) that both the Markov parameters $\mathbf{M}_{\mathbf{i}}$ and covariance parameters $R_{j}$ are known only to be acsurate up to a specified wordlength, and any higher precisional representation is regarded as uncorrelated random noise. Then the calculation of all q-Markov COVERs (for a particular row unitary matrix $U_{q}$ ) will also only be accurate to a finite precision beyond which the parameter representation contains uncorrelated random noise.

Lemma 4.1 Suppose $M=M^{*}>0$ and $K=K^{*}>0$ are given nxn matrices. Let $v_{j} \in R^{n}$ be a zero mean random variable uniformly distibuted between \pm 1 with uncorrelated components which are also uncorrelated with components of $\mathbf{v}_{\mathbf{i}}$. Then we have

$$
E\left\{\mathrm{v}_{\mathrm{j}}^{*} \mathrm{Mv} \mathrm{v}_{\mathrm{j}}\right\}=\frac{1}{3} \mathrm{tr}[\mathrm{M}]
$$

Furthermore

$$
E\left\{\operatorname{tr}\left[\mathrm{V}^{*} \mathrm{MVK}\right]\right\}=\frac{1}{3} \operatorname{tr}[\mathrm{MK}]
$$

where

$$
V=\left[v_{1} v_{2} \cdots v_{n}\right] \varepsilon R^{n \times n}
$$

Unfortunately these results cannot be applied directly to (2.9) since $\mathrm{X}$ itself is a random variable. However if we approximate $\hat{X}$ by $X$ we can deduce the following result.

\section{Theorem 4.1}

Suppose the components of $\delta \mathrm{A}, \delta \mathrm{B}$ and $\delta \mathrm{C}$ are zero mean uncorrelated random variables uniformly distributed between \pm 1 . Then $E[J\}$ where $J=\operatorname{tr}[Y]$ is

$$
875
$$


approximated by $E\{\hat{J}\}$ where

$$
E[\hat{\mathrm{J}}\}=\gamma^{2} \operatorname{tr}\left[\mathrm{B}^{*} \mathrm{~KB}\right]+\left(\gamma^{2}+\frac{\gamma_{0}^{2}}{3}\right) \operatorname{tr}[\mathrm{K}]+\frac{\gamma_{0}^{2}}{3}(\operatorname{tr}[\mathrm{XK}]+\operatorname{tr}[\mathrm{X}])
$$

where $K, X$ are defined by (2.11) and (1.3).

Proof: From (2.9) ignoring the linear term in $\delta C$

$$
\begin{gathered}
\mathrm{J} \simeq \gamma^{2}\left\{\operatorname{tr}[\mathrm{K}]+\operatorname{tr}\left[\mathrm{B}^{*} \mathrm{~KB}\right]\right\}+ \\
\gamma_{0}^{2}\left\{\operatorname{tr}\left[(\delta \mathrm{A})^{*} \mathrm{X}(\delta \mathrm{A}) \mathrm{K}\right]+\operatorname{tr}\left[(\delta \mathrm{B})^{*} \mathrm{~K}(\delta \mathrm{B})\right]+\operatorname{tr}\left[(\delta \mathrm{C})^{*} \mathrm{X} \delta \mathrm{C}\right]\right\}
\end{gathered}
$$

The result then follows using Theorem 2.1.

Under a similarity transformation $\mathrm{T}$, the performance measure (4.5) becomes

$$
E\left\{\hat{\mathrm{J}}_{\mathrm{T}}\right\} \triangleq \gamma^{2} \operatorname{tr}\left[\mathrm{B}^{*} \mathrm{~KB}\right]+\left(\gamma^{2}+\frac{\gamma_{0}^{2}}{3}\right) \operatorname{tr}\left[\mathrm{T}^{*} \mathrm{KT}\right]+\frac{\gamma_{0}^{2}}{3}\left(\operatorname{tr}[\mathrm{XK}]+\operatorname{tr}\left[\mathrm{T}^{-1} \mathrm{X}\left(\mathrm{T}^{-1}\right)^{*}\right]\right.
$$

Note that both $\operatorname{tr}\left[\mathrm{B}^{*} \mathrm{~KB}\right]$ and $\operatorname{tr}[\mathrm{XK}]$ are invariant. In fact the invariant eigenvalues $\left\{\sigma_{k}^{2}\right\}$ of $X K$ are the squares of the Hankel singular values of the system defined by $\{A, B, C\}$. Consequently we need only consider the minimization of

$$
\left(\gamma^{2}+\frac{\gamma_{0}^{2}}{3}\right) \operatorname{tr}\left[\mathrm{T}^{*} \mathrm{KT}\right]+\frac{\gamma_{0}^{2}}{3} \operatorname{tr}\left[\mathrm{T}^{-1} \mathrm{X}\left(\mathrm{T}^{-1}\right)^{*}\right]
$$

over similarity transformations $\mathrm{T}$. We make use of an earlier result [8] to provide the minimum in (4.7).

\section{Theorem $4.2[8]$}

Consider a minimal asymptotically stable order system $\{\mathrm{A}, \mathrm{B}, \mathrm{C}\}$ with controllability grammian $X$ and observability grammian $K$. Let $\tilde{X}$ and $\tilde{K}$ be the transformed grammians as a result of applying a similarity transformation $T$; that is

$$
\tilde{X}=T^{-1} X\left(T^{-1}\right)^{*} ; \tilde{K}=T^{*} K T
$$

Then 


$$
\operatorname{tr}\left[\alpha^{2} \tilde{\mathrm{X}}+\tilde{\mathrm{K}}\right] \geq 2 \alpha \sum_{\mathrm{k}=1}^{\mathrm{n}} \sigma_{\mathrm{k}}
$$

where $\left\{\sigma_{k}^{2}\right\}$ are the Hankel singular values. Moreover equality is achieved in (4.9) if and only if

$$
\tilde{\mathrm{K}}=\alpha^{2} \tilde{\mathrm{X}}
$$

In particular, in (4.7)

$$
\min _{T} E\left\{\hat{J}_{T}\right\}=\gamma^{2} \mathrm{tr}\left[\mathrm{B}^{*} \mathrm{~KB}\right]+\frac{\gamma_{0}^{2}}{3}\left(\sum_{k=1}^{\mathrm{r}_{\mathrm{q}}} \sigma_{\mathrm{k}}^{2}+2 \alpha \sum_{k=1}^{\mathrm{r}_{\mathrm{q}}} J_{\mathrm{k}}\right)
$$

where

$$
\alpha=\sqrt{1+3\left(\gamma / \gamma_{0}\right)^{2}}
$$

The minimum value is achieved in (4.11a) when $\tilde{\mathrm{K}}, \overline{\mathrm{X}}$ satisfy (4.10) with $\alpha$ given by $(4.11 b)$

One optimal realization (4.10) is a scaled internally balanced structure; that is

$$
\tilde{X}_{1}=\alpha^{-1} \operatorname{diag}\left(\sigma_{1}, \sigma_{2}, \ldots, \sigma_{\mathrm{r}_{q}}\right\} ; \tilde{K}_{1}=\alpha \operatorname{diag}\left(\sigma_{1}, \sigma_{2}, \cdots, \sigma_{\mathrm{r}_{q}}\right\}
$$

From the point of view of $1_{2}$-scaling, equal diagonal components of $\tilde{X}$ guarantee equal dynamic range of the state components. It is evident from (4.10) that any unitary transformation $\tilde{U}$ applied to the coordinate basis having $\tilde{\mathbf{X}}$ and $\tilde{\mathrm{K}}$ as the respective controllability and observability grammians will not alter the optimal performance. Consequently an optimal realization in which all diagonal components of the controllability grammian are equal exists with controllability grammian $\tilde{U}^{*} \tilde{X}_{1} \tilde{U}$ and observability grammian $\tilde{U}^{*} \tilde{K}_{1} \tilde{U}$ such that

$$
\tilde{U}^{*} \tilde{X}_{1} \tilde{U}_{j j}=\frac{1}{\alpha r_{q}} \sum_{k=1}^{r_{q}} \sigma_{k} \text { for all } j
$$

where $\tilde{X}_{1}, \tilde{K}_{1}$ are defined by (4.12) and $\tilde{U}$ unitary. The existence of $\tilde{U}$ is guaranteed by lemma 2.1 and an explicit algorithm for constructing a (nonunique) $\tilde{U}$ is available in [9, Appendix A].

\section{Corollary 4.1}

The optimal q-FSWL COVER which minimizes $(2.21)$ subject to the $\mathbf{l}_{2}$ scaling constraint 


$$
\Lambda_{i j}=\frac{1}{\alpha r_{q}} \sum_{k=1}^{r_{q}} \sigma_{k} \text { for all } j
$$

also minimizes $E\left[\hat{\mathrm{J}}_{\mathrm{T}}\right\}$ in (4.6)

This result provides a connection between the optimal q-FSWL COVER structure which minimizes only the effects due to state quantization noise, and the suboptimal q-FWL Markov COVER structure which minimizes $E\left\{\hat{\mathrm{J}}_{\mathrm{T}}\right\}$ subject to the assumed random parameter error model stated in Theorem 4.1. Once again we note that the result is suboptimal in the sense that $\hat{X}$ and $X$ in (2.9) and (4.5) are only approximately equal. The result of Corollary 4.1 is also only of academic value since the $\mathrm{l}_{2}$-constraint (4.14) is not known until the design is complete since the Hankel singular values $\left(\sigma_{j}\right\}$ depend on the optimal row unitary matrix $U_{p}$ as provided in Theorem 2.1. However a more explicit result can be stated.

\section{Corollary 4.2}

The optimal q-FSWL cover subject to the $\mathrm{l}_{2}$-scaling constraint (2.22) also minimizes $E\left\{\hat{\mathrm{J}}_{\mathrm{T}}\right\}$ in (4.12) subject to (2.22).

\section{An Example}

Consider a 5 mode simply supported beam of length $\pi$ having 2 inputs $u_{1}, u_{2}$ and 2 outputs $y_{1}, y_{2}$

$$
\begin{array}{ll}
u_{1}=F(0.2 \pi, t), & u_{2}=T(\pi, t) \\
y_{1}=\theta(0, t), & y_{2}=\mu(0.6 \pi, t)
\end{array}
$$

where $F(0.2 \pi, t)$ denotes a force applied at $.2 \pi$ units from the left end of the beam, $T(\pi, t)$ denotes a torque at the right end of the beam, $\theta(0, t)$ denotes angular deflection at the left end, and $\mu(0.6 \pi, t)$ denotes rectilinear deflection at $0.6 \pi$ from the left end of the beam. The equations of motion are assumed to be described by

$$
\ddot{\eta}_{k}+2 \xi_{k} \omega_{k} \dot{\eta}_{k}+\omega_{k}^{2} \eta_{k}=[\sin (0.2 \pi k) k \cos (\pi k)]\left[\begin{array}{l}
u_{1} \\
u_{2}
\end{array}\right]
$$

\section{8}




$$
\left[\begin{array}{l}
y_{1} \\
y_{2}
\end{array}\right]=\sum_{k=1}^{5}\left[\begin{array}{l}
k \cos (0 \pi k) \\
\sin (0.6 \pi k)
\end{array}\right] \eta_{k}
$$

where $\omega_{k}=k^{2}$ rads $/ \mathrm{sec}$. and $\xi=0.005$. A continuous time 10 th order state space model is defined by

$$
\dot{\mathrm{x}}=\mathrm{Fx}+\mathrm{Gu}, \quad \mathrm{y}=\mathrm{Cx}
$$

where

$$
x=\left(\eta_{1} \dot{\eta}_{1} \eta_{2} \dot{\eta}_{2} \cdots \eta_{5} \dot{\eta}_{5}\right)^{*}
$$

A zero order hold equivalent 10 th order discrete model (1.1) is defined by

$$
A=e^{F T} ; \quad B=\int_{0}^{T} e^{F \sigma} d \sigma G
$$

For the numerical work, a sampling period $\mathrm{T}=0.025 \mathrm{sec}$. was selected which corresponded to approximately 10 samples in the shortest period. The eigenvalues of $A$ are at

$0.996 \pm \mathrm{j} 0.0250,0.9985 \pm \mathrm{j} 0.0500,0.9968 \pm \mathrm{j} 0.0750,0.9945 \pm \mathrm{j} 0.0998,0.9916 \pm \mathrm{j} 0.1246$.

Using the algorithm described in Corollary 1.1 the :ollowing results were obtained.

\begin{tabular}{c|c|c|c|l}
$\mathrm{q}$ & $\mathrm{s}_{\mathrm{q}}$ & $\mathrm{r}_{\mathrm{q}}$ & $\mathrm{p}_{\mathrm{q}}$ & \\
\hline 2 & 2 & 4 & 2 & \\
3 & 2 & 6 & 4 & \multirow{U_{\mathrm{q}}\text{is}2\times2}{}{} \\
4 & 2 & 8 & 6 & \\
5 & 2 & 8 & 8 & \\
6 & 2 & 9 & 9 & no freedom \\
27 & 2 & 10 & 10 & n
\end{tabular}

Hence for $q=2,3,4, U_{q}$ in (1.22b) can be an arbitrary $2 \times 2$ unitary matrix, while for $\mathrm{q} \geq 5$ there is no remaining freedom in the q-COVER.

Optimal q-FSWL COVER designs:

$$
U_{q}=\left[\begin{array}{cc}
\cos \theta_{q} & \sin \theta_{q} \\
-\sin \theta_{q} & \cos \theta_{q}
\end{array}\right] ; \quad \begin{aligned}
& \theta_{2}=40^{\circ} \\
& \theta_{3}=0^{\circ} \\
& \theta_{4}=65^{\circ}
\end{aligned}
$$

(other cases $\left[\begin{array}{cc}1 & 0 \\ 0 & -1\end{array}\right]$ and $\left[\begin{array}{cc}-1 & 0 \\ 0 & 1\end{array}\right]$ were also checked and neither was optimal). 
The cost ranges from (2.29) for $\alpha=1$ were

$$
\begin{aligned}
& \eta_{2 \text { opt }}=0.3143 \times 10^{6} \leq \eta_{2} \leq 0.8478 \times 10^{6} \\
& \eta_{3 \text { opt }}=0.2570 \times 10^{6} \leq \eta_{3} \leq 0.4764 \times 10^{6} \\
& \eta_{\text {Lopt }}=0.0019 \times 10^{8} \leq \eta_{4} \leq 0.1308 \times 10^{8}
\end{aligned}
$$

The actual FWL output roundoff noise is given by

$$
\gamma^{2} \eta_{q} ; \gamma^{2}=\frac{1}{12} 2^{-2 w}
$$

where $\mathrm{W}$ bits are assigned to the fractional wordlength of the state. Hence a factor of 4 improvement in $\eta_{q}$ corresponds to a wordlength saving of 1 bit. There is little savings in this example when $q=2,3$. However for $q=4$ we have a saving of 4 bits. In practice, for fast sampling and low structural damping, the savings would increase as the dimension of the model increases (e.g. a simply supported beam of 50 modes with $q=8$ ). 


\section{References}

[1] D. Williamson, "Structural State Space Sensitivity in Linear Systems," Systems and Control Lett., 7, July (1986) pp. 301-307.

[2] D. Williamson, "Roundoff Noise Minimization and Pole-Zero Sensitivity in Fixed Point Digital Filters using Residue Feedhack," IEEE Trans. on Acoustics, Speech and Signal Processing, Vol. ASSP-34, No. 4, Aug. 1986, pp. 1013-1016.

[3] A.M. King, V.B. Desai and R.E. Skelton, "A generalized approach to qMarkov covariance equivalent realizations for discrete systems," 1987 $A C C$, Minneapolis, USA.

[4] R.E. Skelton and B.D.O. Anderson, "q-Markov Equivalent Realizations," Int.J. Control, Vol. 44, No. 5, 1986, pp. 1477-1490.

[5] R.E. Skelton and E.G. Collins, "Set of q-Markov covariance equivalent models of discrete systems," Int. J. Control, (to appear).

[6] A.B. Stripad and D.L. Snyder, "A necessary and sufficient condition for quantization errors to be uniform and white," IEEE Trans. Acoust. Speech Signal Process, Vol. 25, 1977, pp. 442-448.

[7] B.D.O. Anderson and R.E. Skelton, "The generation of all q-Markov covers," IEEE Trans or Circuits \& Systems (to appear). Also see IFAC Congress, Munich, 1987.

[8] S.Y. Hwang, "Minimum uncorrelated unit noise in state space digital filtering," IEEE Trans. Acoust., Speech, Signal Prosessing, vol. ASSP-25, pp. 273-281, Aug. 1977.

[9] C.T. Mullis and R.A. Roberts, "Synthesis of Minimum Roundoff Noise in Fixed Point Digital Filters," IEEE Circuits and Systems, CAS-23, Sept. 1976, pp. 256-262.

[10] D. Williamson, "Finite state wordlength compensation in digital Kalman filters," IEEE Trans. Auto. Control, Vol. AC-30, No. 10, Oct. 1985, pp. 930-939.

[11] D. Williamson and K. Kadiman, "Finite wordlength linear quadratic Gaussian regulator," Int. Symp. Circuits \& Systems, Philadelphia, USA, June 1987. 